\title{
Recaída en el abandono del consumo de tabaco: una revisión
}

\author{
Quesada, M.; Carreras, J.M.; Sánchez, L.
}

Unidad de Tabaquismo. Servicio de Neumología. Hospital Carlos III.

Instituto de Salud Carlos III. Psicóloga - Médico adjunto - Jefe del Servicio de Neumología

\section{RESUMEN}

La recaída constituye un evento frecuente en el proceso de dejar de fumar siendo, en parte, responsable de la alta prevalencia de tabaquismo. El objetivo del presente trabajo es revisar y comentar los diferentes enfoques teóricos y los factores determinantes de este proceso tanto a nivel individual como ambiental, así como los principales procedimientos utilizados en la prevención de recaídas según el análisis de la bibliografía científica publicada en los últimos 20 años. Los estados emocionales negativos, los altos niveles de dependencia y el escaso grado de apoyo social son variables claramente relacionadas con este proceso en diferentes poblaciones incluyendo la población española. Intensificar los programas de tratamiento, entrenar en habilidades para afrontar las distintas situaciones de riesgo y el control de los factores emocionales así como fomentar la búsqueda de apoyo social son formas de intervención que se han mostrado eficaces para la prevención de la recaída en el abandono del consumo de tabaco.

Palabras Clave. tabaquismo, recaída, tratamiento, factores individuales, factores ambientales, prevención. dientes.

\section{SUMMARY}

Relapse is an usual event in smoking cessation and it has a part of responsibility in the smoking high prevalence. The objective of this paper is to review and comment the several theoretical models, individual and environmental relapse determinant factors and principal proceedings for relapse prevention through the analysis of the past twenty years scientific bibliography. Emotional states, high dependence and low social support degree are variables clearly related with this process in different populations including spanish population. Intensify treatment programs, skill training to cope with risk situations and to control emotional factors and encourage social support search are intervention ways that have shown as efficacious for smoking cessation relapse prevention.

Key words: smoking, relapse, treatment, individual factors, environmental factors

\section{INTRODUCCION}

$\mathbf{E}$ I consumo de cigarrillos constituye la principal causa aislada y evitable de morbi-mortalidad en España, originando más de 46.000 muertes al año; una de cada cuatro muertes producidas en hombres y una de cada cincuenta producidas en mujeres, lo que representa el $14 \%$ de la mortalidad general prematura (González, et. al., 1997).

La mitad de las personas que fuman van a morir a consecuencia de alguna enfermedad atribuible al tabaco y el $50 \%$ lo hará durante su vida activa perdiendo entre 20 y 25 años de vida (Peto, et. al., 1992). Las personas que dejan de fumar viven más tiempo y con una mejor calidad de vida que las personas que siguen fumando, alcanzándose estos beneficios a cualquier edad en que se abandone el consumo (USDHHS, 1990; CDC, 1991).

El descenso en la prevalencia del consumo de tabaco se está produciendo lentamente habiendo pasado la proporción de fumadores entre la población mayor de 16 años de un $38.1 \%$ en 1987 a un 36,9\% en 1995 y a un 35,7\% en 1997 (Nieto, 2000). El 70\% de los fumadores quiere dejar de fumar (USDHHS, 1990) pero menos del $10 \%$ alcanza la abstinencia al cabo de un año (Fiore, et.al. ,1990) ya que el porcentaje de recaídas es muy elevado al igual que en otras dependencias. Se estima que entre un $50 \%$ y un 
$75 \%$ de los fumadores que han seguido un programa de tratamiento recaen en el año siguiente (Glasgow y Lichtenstein, 1987) incrementándose estos porcentajes en aquellos que abandonan el consumo de forma espontánea (USDHHS; 1990). La mayoría de las recaídas se producen en los tres primeros meses posteriores al abandono (Echeburúa y De Corral, 1986; Garvey et.al., 1992; Gilpin, Pierce y Farkas, 1997) y muchos fumadores van a requerir varios intentos antes de alcanzar la abstinencia consolidada (Rose, 1996). El considerar la recaída como parte del proceso de dejar de fumar es por tanto fundamental al abordar este tipo de adicción.

El análisis de las recaídas es actualmente objeto de diversos estudios, centrándose tanto en sus factores determinantes como en el desarrollo de programas de prevención que potencian la efectividad de los tratamientos.

En nuestro país, en los últimos años, se están llevando a cabo estudios dirigidos a evaluar técnicas y programas de tratamiento del tabaquismo pero son escasos los que analizan el proceso y las causas de la recaída por lo que hemos creído oportuna esta revisión que aporta una exhaustivo análisis de la bibliografía científica sobre el tema incluyendo trabajos españoles.

El objetivo por tanto del presente trabajo es revisar los diferentes enfoques teóricos, los determinantes del proceso de recaída y los factores predictores del éxito en el abandono del consumo de tabaco, así como los principales procedimientos utilizados en la prevención de recaídas.

\section{DEFINICIONY MODELOS}

La definición de la recaída va a estar en condicionada por el concepto de abstinencia y por las diferentes formas de medir el estatus del fumador.

- Abstinencia: Los procedimientos habituales de evaluar la abstinencia son:

Autoinforme: Información que nos da la persona considerándose las siguientes formas de medida (Velicer, et. al., 1992):

- Prevalencia de abstinencia puntual: refleja la ausencia de consumo de tabaco en un momento puntual en el tiempo. Los periodos mínimos de abstinencia que más se han utilizado han sido de 24 horas, 7 días y 30 días. Cuando el periodo de abstinencia es de una semana o menos presenta la ventaja de que puede ser validada a través de mediciones biológicas (Benowitz, 1983).

- Abstinencia continua: Ausencia continuada de consumo de tabaco desde la realización de algún tipo de tratamiento o algún evento significativo.

- Abstinencia prolongada: se considera abstinentes prolongados a aquellas personas que no han fumado en un periodo prolongado de tiempo (6-12 meses) aunque el inicio no coincida con una intervención o evento significativo.

Medidas Bioquímicas: El humo del cigarrillo se compone de diferentes agentes químicos, algunos propios del tabaco y otros que se generan durante el proceso de fumar (USDHHS, 1990). La abstinencia puede ser también constatada por la presencia o no de estas sustancias en el organismo. La técnica más utilizada ha sido la medición de monóxido de Carbono (CO) cuya valoración se puede hacer directamente a través de aire espirado o haciendo una medición del porcentaje de hemoglobina combinada con $\mathrm{CO}$ : carboxihemoglobina ( $\mathrm{COHb}$ ). Los valores de $\mathrm{CO}$ en aire expirado para determinar si el sujeto fuma o no oscilan entre 5 y 10 ppmm y son una medida fiable de la abstinencia si se tiene en cuenta que el CO tiene una vida corta (entre 2 y 5 horas) (Jarvis, Russell y Salojee, 1980). La determinación de $\mathrm{COHb}$ se puede realizar a través de un examen sanguíneo siendo el punto de corte para considerar al sujeto fumador de 1,77\% (percentil 95) (Janzol, et.al. , 1981). Otro marcador es el tiocianato. Presenta una vida media de entre 10 y 14 días y su forma de medida más usual es a través de la saliva considerándose el punto de corte en los $100 \mathrm{mg}$. Sin embargo, es poco específico ya que existe un numero importante de alimentos que lo contienen. La cotinina es otro marcador biológico utilizado. Principal producto de degradación de la nicotina persiste en el organismo hasta cuatro días desde que la persona deja de fumar y puede medirse en saliva, sangre y orina. El punto de corte se encuentra en 200-400 ng/ml (Haley, Axelrad y Tilton, 1983).

- Desliz: Para Ossip-Klein y colaboradores (1986) un desliz es un episodio de no más de una semana de duración en el que el sujeto fuma al menos una calada al día después haber permanecido abstinente al menos 24 horas.

- Recaída: Según los autores anteriores la recaída se produce cuando el patrón de consumo descrito se mantiene durante más de 7 días consecutivos. Otras investigaciones, sin embargo, insisten en la necesidad de considerar en la definición de recaída 
tanto el factor duración como el factor de tasa de consumo. Así Shiffman y colaboradores en 1996 proporcionan una definición alternativa en la que incluyen las dos variables: la recaída se produce cuando después de un periodo de abstinencia de al menos 24 horas se consumen cinco o más cigarrillos al día durante tres días consecutivos (Shiffman, et.al., 1996b).

Los modelos explicativos de las recaídas se centran en los que analizan el proceso de la recaída y los mecanismos que la desencadenan:

\subsection{Modelos del proceso de recaída}

Se han descrito tres modelos del proceso de recaída: acumulativo, episódico e interactivo. Aunque difieren entre sí todos conceden especial importancia a las fluctuaciones a través del tiempo de la predisposición a la recaída (PR). La PR se define como el riesgo a recaer en un momento determinado (Ossip-klein, et.al. , 1986)

2.1.1. Modelo acumulativo: Propone que los cambios en la PR se producen de forma continua. Concede especial relevancia a determinadas variables predisponentes que tendrían un impacto acumulativo. Por ejemplo la recaída puede ocurrir cuando los niveles de estrés superan un determinado umbral. Enfatizan el estudio basal de estas variables. En este modelo se basan los estudios que predicen la recaída a través de las variables de personalidad (Ockene, et. al., 1982) o que exponen los altos porcentajes de recaídas en los fumadores con elevados niveles de dependencia (Graham y Gibson, 1971).

2.1.2. Modelo episódico: Considera la PR como estable hasta que un evento repentino precipita la recaída. El cese de la abstinencia puede ocurrir como consecuencia de que este evento haga que la PR supere un determinado umbral. Está implícito en los estudios que analizan los episodios de recaída. (Marlatt, y Gordon, 1985; Shiffman, 1986; Baer y Lichtenstein, 1988).

2.1.3. Modelo interactivo: Representa una combinación de los modelos anteriores. Como el modelo episódico admite que la recaída es precipitada por un evento agudo que lleva a la PR a superar un determinado umbral. A su vez admite cambios continuos en la PR como antecedentes fundamentales para que junto con los eventos puntuales se produzca la recaída. Hay escasez de trabajos empíricos que utilicen este modelo.(Ossip-Klein, et.al., 1986)

\subsection{Modelos de los determinantes de la de recaída}

Originados por las variables que producen cambios en la predisposición a la recaída. Los mecanismos o modelos de recaída que se describen a continuación (Tabla 1.) no son incompatibles con los descritos anteriormente sino que incorporan sus postulados, diferenciándose por tanto en el énfasis que ponen en unas $u$ otras variables.

2.2.1. Modelo de Aprendizaje Social: Basado en el Teoría del Aprendizaje Social (Bandura, 1986) propone una asociación entre las características de la persona (creencias y expectativas sobre el mantenimiento de la abstinencia, grado de dependencia física), la conducta (experiencias previas de abandono) y el ambiente (eventos estresantes, normas sobre restricciones de consumo). Las variables ambientales pueden incluir también características comunitarias como la percepción de la normativa respecto al tabaco y las restricciones aunque éstos factores no han sido suficientemente estudiados como predisponentes de la recaída (Curry, et.al. , 1993).

2.2.2. Modelo Bioconductual: La recaída se puede producir por diferentes efectos biológicos: efectos biológicos del condicionamiento directo (situaciones asociadas al consumo pueden elicitar los efectos del mismo)(Grunberg y Baum, 1985); efectos biológicos del contracondicionamiento (estímulos asociados al consumo de tabaco pueden provocar efectos contrarios al mismo incrementándose la posibilidad de un desliz) (Solomon y Corbit, 1973) y los efectos propios de la abstinencia (O’Conell y Martin, 1987).

2.2.3. Modelos de Estado: (Prochaska y Di Clemente, 1983) La conducta de dejar de fumar se considera como un proceso en el que la persona pasa por diferentes estadios. La recaída será un estadio más del proceso y se produce cuando las estrategias de estabilización y mantenimiento fallan.

2.2.4. Modelo de afrontamiento del estrés: Según el modelo de Marlatt y Gordon (1980) existen determinadas situaciones (situaciones de alto riesgo) en las que es mayor la probabilidad de que se produzca la recaída. Que finalmente se produzca o no va a depender del modo en que la persona se enfrente a la situación. La falta de habilidades de afrontamiento, situaciones de estrés o el consumo de otras sustancias como el alcohol pueden llevar a un fallo en el afrontamiento de dicha situación. Como consecuencia de esto disminuye la autoeficacia y aumentan las expectativas positivas sobre el consumo lo que facilitaría un primer desliz. La posibilidad de que el consumo continúe hasta una recaída dependerá de la consecuente reacción cognitivo-afectiva (Efecto de Violación de la Abstinencia). 
Tabla 1: Modelos de los mecanismos de recaída en el proceso de dejar de fumar.

\begin{tabular}{|c|c|c|c|}
\hline Modelo & Autores & Base & Explicación recaída \\
\hline $\begin{array}{l}\text { Modelo de } \\
\text { aprenidizaje social. }\end{array}$ & Bandura, 1986. & $\begin{array}{l}\text { Influencia del aprendizaje social. } \\
\text { Interacción conducta, variables } \\
\text { personales y medio. }\end{array}$ & $\begin{array}{l}\text { La presencia y frecuencia de } \\
\text { estímulos ambientales } \\
\text { elicitadores de la conducta de } \\
\text { fumar. }\end{array}$ \\
\hline $\begin{array}{l}\text { Modelo } \\
\text { bioconductual. }\end{array}$ & Wise, 1988. & $\begin{array}{l}\text { Mecanismos neuronales } \\
\text { reforzamiento positivo y negativo. }\end{array}$ & Deseo intenso de fumar o craving. \\
\hline Modelo de estado. & $\begin{array}{l}\text { Prochaska y Di } \\
\text { Clemente } 1983 .\end{array}$ & $\begin{array}{l}\text { Estadios de cambio en el proceso } \\
\text { de dejar de fumar (precontemplación, } \\
\text { contemplación, acción, mantenimiento } \\
\text { y/o recaída). }\end{array}$ & $\begin{array}{l}\text { La recaída es un estadio más en } \\
\text { el proceso de dejar de fumar. }\end{array}$ \\
\hline $\begin{array}{l}\text { Modelo de } \\
\text { afrontamiento } \\
\text { del estrés. }\end{array}$ & Marlatt y Gordon, 1980. & $\begin{array}{l}\text { La conducta de fumar como estrategia } \\
\text { de afrontamiento del estrés. } \\
\text { Percepción de falta de habilidades. }\end{array}$ & $\begin{array}{l}\text { Ante situaciones de alto riesgo y } \\
\text { percepción de falta de habilidades } \\
\text { para afrontarlas. (efecto de viola } \\
\text { ción de la abstinencia). }\end{array}$ \\
\hline
\end{tabular}

\section{PROCESO DE RECAIDA: FACTORES DETERMI- NANTES}

La recaída no es un hecho que se produzca de forma espontánea sino que forma parte de un proceso en el que intervienen múltiples factores. El estudio de los determinantes de la misma se plantea, por tanto, como un tema fundamental. Desde los primeros intentos por clasificar los episodios de recaída (Marlatt y Gordon, 1980; Cummings, Gordon y Marlatt, 1980) ha existido un interés general en la investigación por describir tanto los factores individuales como las situaciones que se relacionan con el mantenimiento o no de la abstinencia al dejar de fumar y de cómo estas variables pueden funcionar como predisponentes o como precipitantes en el proceso de recaída. La Tabla 2 muestra las variables más estudiadas.

\subsection{FACTORES INDIVIDUALES}

\subsubsection{Sociodemográficos}

Las variables sociodemográficas se consideran condicionantes del éxito del abandono del consumo de tabaco. Los resultados de su análisis no son homogéneos y están condicionados por distintos factores como las características de la muestra en la que se realiza el estudio (los resultados varían de un país a otro) o si las personas siguen o no un tratamiento para dejar de fumar.
Las variables objeto de mayor investigación han sido edad, género, nivel socioeconómico y educativo.

Nides y colaboradores (1995) en un grupo de 3.923 fumadores que había realizado tratamiento cognitivo conductual en grupo, encuentran que el sexo masculino y tener un nivel de educación alto son factores predictores de éxito. En nuestro país Moreno y Herrero (2000) presentan resultados similares. Hymowitz y colaboradores (1997) en una muestra de fumadores que espontáneamente dejan de fumar concluye que los hombres de más edad tienen mayor probabilidad de lograr la abstinencia.

Otros investigaciones como las de Whitlock y colaboradores (1997), Faue y colaboradores (1997) y Matheny y Weatherman (1998), sin embargo no encuentran estas diferencias al analizar a fumadores americanos sometidos a tratamiento.

\subsubsection{Emocionales}

Los estados de ansiedad, irritabilidad, estrés y/o depresivos están vinculados a episodios de recaídas.

Inicialmente tanto Marlatt y Gordon (1980) que dividen en dos categorías los factores que determinan estos episodios (determinantes intrapersonalesambientales,/determinantes interpersonales), como Shiffman $(1982,1986)$ mediante análisis multivariante (Tabla 2) encontraron que los estados emocionales negativos podían condicionar los episodios de recaída. Estos resultados han sido reproducidos posteriormente en numerosos estudios (O'Conell y Martin, 1987; 
Baer y Lichtenstein, 1988; Becoña, Lista y Frojan, 1989; Bliss, et.al.,1989; O'Conell, 1990).

Drobes, Meier y Tiffany (1994) exponen a 60 fumadores que han realizado un programa para dejar de fumar (utilizando técnicas de fumar rápido y entrenamiento en habilidades de afrontamiento) a situaciones imaginarias de alto riesgo y encuentran que al incorporar a las situaciones elementos relacionados con emociones negativas (como por ejemplo el estrés producido por algún problema doméstico), los sujetos se confiesan menos capaces de afrontarlas sin fumar. Concluyen que la capacidad para mantener la abstinencia se ve claramente disminuida bajo estados emocionales negativos.

Más recientemente Shiffman y colaboradores (1996a) han llevado a cabo una investigación en tiempo real con 108 exfumadores que habían finalizado un tratamiento cognitivo-conductual en grupo y que llevaban al menos 24 horas sin fumar. Analizan las primeras tentaciones y deslices, y observan que las personas se sienten peor cuando se produce un desliz que cuando sólo se han sentido tentados. El 62\% de los participantes afirma que cuando fumaron por primera vez después del periodo de abstinencia, se encontraban bajo estados emocionales altamente negativos, principalmente de estrés e ira. Concluyen que el hecho de fumar puede deberse a un intento por parte de la persona de controlar estados de ánimo negativos.

Las personas depresivas fuman en una gran proporción, presentan una importante dependencia a la nicotina y son menos capaces de dejar de fumar que aquellas que no tienen historia de depresión. Los fumadores con episodios previos de depresión tienen más riesgo de presentar síntomas depresivos durante el síndrome de abstinencia pudiendo ser motivo de recaída (Glassman et al 1990; Anda et al 1990; Covey, Glassman y Stetner,1997). Se ha especulado que los sujetos depresivos pueden utilizar la nicotina como una forma de automedicación para prevenir la aparición de episodios depresivos. (Hughes, 1988). Vázquez y Becoña (1998) en una revisión de las publicaciones sobre este tema concluyen que dejar de fumar es más difícil para el fumador deprimido que para el resto de la población, pues presenta síntomas de abstinencia más intensos (irritabilidad, ansiedad y disforia) teniendo mayor probabilidad de fracasar en su intento (Vázquez y Becoña, 1998).

\subsubsection{Afrontamiento}

La utilización de estrategias de afrontamiento ante las situaciones de alto riesgo disminuye la probabilidad de que se produzca una recaída (Kamark y Lichtenstein,1988; Baer, et.al.,1989; Shiffman et al, 1996 a; O’Conell et al 1998).
Se diferencian principalmente estrategias cognitivas y estrategias conductuales. De entre las primeras, se han descrito como más frecuentes el pensar en las consecuencias positivas de no fumar y en las negativas de seguir fumando, la distracción cognitiva y las autoinstrucciones. Respecto a las conductuales se encuentra el consumir comida o bebida, retrasar el momento, escapar de la situación y utilizar la relajación. (Shiffman, 1986; Bliss et al, 1989). La mayoría de los autores coinciden en que ambas estrategias son igualmente efectivas, (Curry y Marlatt, 1985; Kamarck y Lichtenstein, 1988; Baer et. al. , 1989) aunque Shiffman (1996 a) encuentra que en el 94\% de las ocasiones en que la situación de alto riego había sido superada el sujeto había utilizado un afrontamiento cognitivo.

Sin embargo, los intentos por establecer este tipo de variables como predictoras o no de recaída han fracasado. Es cierto que cuando el sujeto supera la situación con éxito normalmente ha utilizado algún tipo de afrontamiento, pero esto no significa que no pueda recaer. Se ha propuesto que la efectividad del afrontamiento depende, no sólo del tipo de estrategia utilizada, si no de la rapidez y convicción con que la persona la lleve a cabo. (Baer et al, 1989; Shiffman et. al., 1996a)

\subsubsection{Fisiológicos}

El Tabaquismo se define como una drogodependencia, siendo la nicotina una sustancia psicoactiva con acciones específicas sobre receptores del sistema nervioso distribuidos con mayor densidad en un conjunto de núcleos vinculados con el sistema de recompensa (sistema mesocorticolímbico) y cuyo estímulo repetido ocasiona un fenómeno de adaptación neuroendocrina en el que incluso aumenta el número de dichos receptores en determinadas zonas (Benwell, Balfour y Anderson, 1988). Al cesar su aporte se ocasiona un típico síndrome de abstinencia que forma parte de los criterios diagnósticos de esta dependencia (APA, 1994) y se caracteriza, entre otros síntomas, por la aparición de irritabilidad, disforia, ansiedad, dificultad de concentración y deseo intenso de fumar (craving), alcanzando su nivel más alto de intensidad a las 24-72 horas de haber abandonado el consumo. La nicotina es una de las drogas más adictivas (Hughes, 1992), siendo el deseo uno de los síntomas más persistentes tras el abandono del consumo. (Hughes, 1994).

Se ha propuesto que las recaídas que se producen en los primeros días son atribuibles a niveles altos de deseo y otros síntomas de abstinencia ( $O^{\prime}$ Conell y Martin, 1987). En esta línea, Piasecki, Fiore y Baker (1998) realizaron un estudio para observar la relación del síndrome de abstinencia con las recaídas. Analizaron los síntomas de abstinencia de dos estudios clíni- 
cos hechos con parches de nicotina y encontraron que algunos fumadores presentan patrones atípicos en la sintomatología, mostrándose como predictores de recaída tanto la duración como la intensidad de los síntomas de abstinencia. Sugieren que la persistencia de los síntomas de abstinencia tiene un efecto negativo en la motivación de los pacientes para continuar abstinentes lo que contribuye a la aparición de estados de ánimo negativos. Killen y Fortmann (1997) encontraron que de 2600 sujetos estudiados el 32\% de los que experimentaban elevados niveles de deseo recaían a la semana de haber dejado de fumar.

Shiffman y colaboradores (1997) analizan las características individuales que diferencian a los sujetos que se mantienen abstinentes de los que recaen, en 105 sujetos que llevaban al menos 24 horas sin fumar realizando tratamiento cognitivo-conductal en grupo, los cuales registraban los episodios de consumo en tiempo real durante las siguientes cuatro semanas. Encuentran que los sujetos con mayores niveles de dependencia (medida con el test de Fagerström) recaían más cuando se encontraban bajo estados emocionales negativos y/o niveles intensos de deseo. Otros autores también han encontrado una relación positiva entre niveles elevados de dependencia, niveles intensos de deseo y mayor número de recaídas.( Hellman, et.al. , 1991;Killen, Fortmann, et.al. , 1992; Swan, Ward y Jack, 1996)

Aunque existe clara evidencia de que determinados factores fisiológicos influyen en el mantenimiento de la abstinencia, sobre todo a corto plazo, algunas cuestiones requieren más atención como son los procesos por los cuales los síntomas de abstinencia, y principalmente el deseo, podrían estar influyendo en la aparición de recaídas y su relación con los estados de ánimo negativos.

\subsubsection{Historia de consumo de la sustancia.}

Las variables de forma de consumo, años de fumador, intentos previos de abandono, número de cigarrillos día, edad de inicio y tiempo transcurrido desde el momento de levantarse y fumar el primer cigarrillo, se han relacionado con las recaídas. Hymowitz y colaboradores (1997) tras realizar un seguimiento telefónico a 13425 fumadores durante cinco años encuentran que entre los mejores predictores de abandono del consumo se encuentran el fumar menos cigarrillos al día, mayor tiempo transcurrido desde el momento de levantase y fumar el primer cigarrillo, empezar a fumar después de los 20 años y haber realizado algún intento serio previo de abandono. Este estudio está en la línea de otras investigaciones que llegan a resultados similares (Ockene , 1982; Cohen et al. , 1989; Castillo y Musitu, 1992; Nides et. al. , 1995; Ockene et. al., 2000). Más específicamente relacionado con los tratamientos de tabaquismo, se ha observado que un fac- tor predictor de éxito a los seis meses es el no haber tenido ni un desliz en todo el proceso (Sánchez, Carreras, Maldonado, 1998; Moreno y Herrero, 2000).

Las conclusiones de estas investigaciones deben ser consideradas a la hora de enfocar los diferentes abordajes del problema del tabaquismo. Las variables relacionadas con el hábito de consumo y la dependencia deben tenerse en cuenta e incluir en los tratamientos de esta adicción medidas que ayuden a paliar los efectos de la abstinencia a la nicotina (p.ej. terapias sustitutivas con nicotina, bupropion).

\subsubsection{Motivacionales}

Los estudios sobre predictores de éxito en el abandono del consumo del tabaco también tienen en cuenta el análisis de los motivos que alegan las personas para dejar de fumar. De esta forma, el estudio anteriormente comentado de Hymowitz encuentra que el $90.2 \%$ de las personas que dejaron de fumar referían motivos de salud, seguidos de un $52.8 \%$ que alegaba el gasto económico y un $52.4 \%$ cuya motivación era dar buen ejemplo. Sin embargo, estos autores no encuentran diferencias significativas entre los motivos de las personas que abandonaron el consumo con éxito y las que continuaron fumando. Esta relación si ha sido confirmada en otro estudio (Halpern y Warner, 1993) en el que se analizaron las razones para dejar de fumar y su asociación con el éxito en el abandono en una encuesta realizada a 7700 personas. Los autores encontraron que el éxito del abandono estaba relacionado con motivos de salud y ser ejemplo para los hijos, mientras que los referidos al coste económico, al efecto del tabaco en otras personas y a la presión familiar se relacionaban con la disminución del consumo pero no con la cesación total.

Los aspectos motivacionales van a ser fundamentales para que los fumadores en fase de precontemplación avancen en el proceso de dejar de fumar.

\subsubsection{Otros factores}

La ganancia de peso tras el abandono del consumo de tabaco y el alto porcentaje de recaídas tras el embarazo generan un especial interés en la literatura científica actual.

Es un hecho constatado que al dejar de fumar se produce un aumento del peso, que oscila entre 2 y 6 kilos al año de abstinencia. La ganancia de peso ocurre en un primer periodo volviendo a recuperar la mayoría de las personas el peso previo sin necesidad de realizar un esfuerzo excesivo. (Hultquist, Ray y Sadish, 1997; Klesges, et al, 1997; Mizoue,et.al.,1998; O’Hara, et al, 1998). Sin embargo, tanto la ganancia de peso como la preocupación previa por que esto suceda influyen negativamente en los diferentes momen- 
tos del proceso: decisión de dejar de fumar, momento de dejarlo, mantenimiento de la abstinencia y recaída. Además estas variables han mostrado diferencias de género significativas, apareciendo más frecuentemente en mujeres (Chen et. al., 1998; Ockene et al, 2000). En un estudio realizado con 580 participantes que habían llevado a cabo un tratamiento para dejar de fumar (basado en reducción gradual de cigarrillos y estrategias de prevención de recaídas) se observó que las personas más preocupadas por el peso eran mujeres, con peso normal o por debajo de lo normal y que normalmente hacían dietas. Este grupo presentó menor porcentaje de abstinencia al final del tratamiento y durante el seguimiento (1-6-12 meses) que el grupo de personas poco preocupadas por el peso. (Meyers, et al. 1997)

Aunque muchas mujeres dejan de fumar durante el embarazo, bastantes vuelven a fumar poco tiempo después de dar a luz. La preocupación por la salud del niño es el principal motivo del abandono del consumo, por lo que en muchas ocasiones la abstinencia permanece durante el tiempo de lactancia. Las variables que más se han relacionado con el retorno al consumo de tabaco han sido que la pareja sea fumadora, que la abstinencia no haya sido absoluta durante el embarazo, la presencia de fumadores en el entorno inmediato (p.ej. las primeras visitas después del parto) y el escaso nivel de confianza en mantenerse abstinentes después de dar a luz (Mullen, et.al.,1997; Edwards y Sims-Jones, 1998; Ratner, et.al, 2000). Con programas específicos para embarazadas se han obtenido buenos resultados, sin embargo se ha observado que la abstinencia no se mantiene a largo plazo (SeckerWalker, et al. 1995), por lo que se ha expuesto la necesidad de desarrollar programas de deshabituación que no se dirijan exclusivamente al periodo del embarazo o lactancia sino que promuevan estrategias de mantenimiento de la abstinencia a largo plazo incidiendo en otro tipo de motivaciones más personales y duraderas (salud, dependencia) (McBride, et al. 1999; Johnson, et.al. , 2000; Ratner, et al. 2000)

Tabla 2: Variables relacionadas con el proceso de recaída (adaptada de Shiffman, et. al., 1986)

\begin{tabular}{|c|c|c|}
\hline \multirow[b]{2}{*}{ DEMOGRÁFICOS } & FACTORES PREDISPONENTES & FACTORES PRECIPITANTES \\
\hline & $\begin{array}{l}\text { Edad } \\
\text { Sexo } \\
\text { Profesión } \\
\text { Nivel socioeconómico } \\
\text { Nivel educativo } \\
\text { Raza } \\
\text { Estado civil } \\
\end{array}$ & Cambios \\
\hline PERSONALES & $\begin{array}{l}\text { Habilidades de afrontamiento } \\
\text { Estilos cognitivos } \\
\text { Actitudes } \\
\text { Factores de personalidad } \\
\text { Estatus de salud } \\
\text { Consumo de otras sustancias } \\
\text { Estado de ánimo }\end{array}$ & $\begin{array}{l}\text { Afrontamiento } \\
\text { Estado de ánimo } \\
\text { Estado de ánimo } \\
\text { Consumo de otras sustanacias }\end{array}$ \\
\hline HISTORIA DE CONSUMO & $\begin{array}{l}\text { Años } \\
\text { Marca/Dosis } \\
\text { Intentos previos de abandono } \\
\text { Motivos para fumar } \\
\text { Motivos para no fumar } \\
\text { Recaídas previas }\end{array}$ & $\begin{array}{l}\text { Motivos/Incentivos } \\
\text { Estado } \\
\text { Deslices } \\
\text { Consumo de otros productos } \\
\text { de tabaco }\end{array}$ \\
\hline BIOLOGICOS & $\begin{array}{l}\text { Dependencia } \\
\text { Genética } \\
\text { Efectos del consumo en el organismo } \\
\text { Reactividad } \\
\text { Factores bioquímicos y endocrinológicos } \\
\text { Síntomas de abstinencia } \\
\text { Peso }\end{array}$ & $\begin{array}{l}\text { Síntomas de abstinencia } \\
\text { Efectos del abandono } \\
\text { Reactividad } \\
\text { Condicionamiento } \\
\text { Tabaquismo pasivo } \\
\text { Peso } \\
\text { Deslices }\end{array}$ \\
\hline AMBIENTALES & $\begin{array}{l}\text { Políticas (ej. Áreas sin humo) } \\
\text { Estímulos relacionados con } \\
\text { la conducta de fumar }\end{array}$ & $\begin{array}{l}\text { Estímulos relacionados con } \\
\text { la conducta de fuma }\end{array}$ \\
\hline SOCIALES & $\begin{array}{l}\text { Apoyo social } \\
\text { Influencia (ej. Presión grupal) } \\
\text { Historia familiar de consumo } \\
\text { Relaciones interpersonales }\end{array}$ & $\begin{array}{l}\text { Apoyo social } \\
\text { Influencia } \\
\text { Relaciones interpersonales }\end{array}$ \\
\hline
\end{tabular}




\subsection{FACTORES AMBIENTALES}

\subsubsection{Situaciones de riesgo}

El término situaciones de alto riesgo se utiliza para deferir aquellas circunstancias en las que existe una mayor probabilidad de que se produzca la recaída. Su identificación puede ser útil de cara al desarrollo de estrategias previas para superar la situación con éxito. Se han analizado las siguientes:

- Situaciones de estrés: Existe evidencia de que las situaciones estresantes están relacionadas con episodios de recaída, incluso se ha observado que durante estos periodos estresantes es más probable que ocurra una recaída que un desliz, (Lichtenstein, et. al,. 1986) y que si el desliz se produce bajo estas circunstancias progresa más rápidamente hacia la recaída que en otras situaciones (Shiffman, et. al., 1996b). Shiffman (1997) observa que casi la mitad de los pacientes $(48 \%)$ refiere haber fumado bajo circunstancias estresantes.

- Situaciones sociales: Gran parte de las situaciones en las que la persona cesa la abstinencia son situaciones sociales. Las más comunes ocurren en tiempo de ocio, en restaurantes o bares y normalmente la persona se encuentra con amigos o personas con las que está manteniendo una relación social. La probabilidad de consumo se incrementa si las otras personas fuman, si en el lugar está permitido fumar o si el tabaco se encuentra disponible. Es muy común que la persona haya comido, haya tomado café y/o haya bebido alcohol previamente. (Merlmestein y Lichtenstein, 1983; Shiffman, 1986; Curry y Mc Bride, 1995; Shiffman, et. al.,1996a; Shiffman, et. al.,1997).

- Otras situaciones: También con cierta frecuencia aparecen tentaciones en situaciones de descanso, en periodos de transición entre una actividad y otra, y en casi la mitad de los casos la persona se encuentra sola, normalmente en casa. Se ha observado escasa probabilidad de que se produzcan deslices mientras la persona está trabajando. A su vez, la probabilidad de que la persona fume en situaciones de impaciencia o desasosiego es significativa. (Shiffman, 1986; Sutton 1992; Shiffman et. al., 1996 a)

El análisis de las situaciones en las que existe mayor probabilidad de que una persona vuelva a fumar tras haber realizado un intento serio de abandono requiere una mayor investigación. Existen algunas preguntas que todavía no están contestadas con claridad como por ejemplo ¿por qué algunas personas recaen en determinadas situaciones y otras no lo hacen en situaciones similares?, ¿qué relación existe entre el superar con éxito o no una situación y determinadas características individuales como por ejemplo los estilos de afrontamiento del sujeto?, ¿existe correlación entre niveles de dependencia y situaciones de riesgo?. Estas y otras cuestiones requieren un mayor análisis lo que contribuiría a la posibilidad de desarrollar entrenamientos más específicos y estrategias individualizadas en las personas que desean superar esta adicción.

\subsubsection{Entorno social}

Existen diferentes investigaciones sobre el papel del apoyo social en el abandono del consumo de taba$c o$, en el mantenimiento de la abstinencia y en la recaída. Las variables más estudiadas han sido:

- el apoyo de la pareja tanto en el momento de dejar de fumar como en el mantenimiento de la abstinencia

- la presencia de fumadores o no fumadores en la red social inmediata de la persona

- la percepción por parte del sujeto de la existencia de fuentes generales de apoyo a los no fumadores.

El apoyo de la pareja se ha mostrado como muy relevante tanto en el momento en que la persona deja de fumar como en el mantenimiento de la abstinencia a largo plazo. Cohen y Lichtenstein (1990) muestran una asociación entre éxito en el abandono y apoyo de la pareja percibido. En otro estudio en el que se entrevistaba telefónicamente a personas que habían dejado espontáneamente de fumar los autores encuentran que la mayor percepción de apoyo por parte de la pareja podía considerarse como una variable predictora de la abstinencia a medio-largo plazo. (Gulliver, et. al., 1995).

La presencia o no de fumadores en el ambiente más inmediato: pareja fumadora, existencia de otros fumadores en la casa y/o en la red social inmediata a la persona es una variable que ha demostrado su validez como predictora de recaída en diferentes trabajos (Wojcik,1988; Bliss, et. al.,1989; Carmody, 1992; Garvey, et. al.,1992; Nides, et. al.,1995).

La importancia del apoyo social ha sido contemplada también a la hora de diseñar tratamientos para dejar de fumar. Al incluir en los programas de tratamiento grupos de apoyo con los que tener un contacto regular los porcentajes de abstinencia mejoran (Gruder, et. al., 1993). Del mismo modo se ha observado que el incluir algún otro tipo de seguimiento, como el que puede realizarse por contacto telefónico, puede ser una estrategia que potencie los efectos de otras intervenciones más intensivas (Lando, et. al., 1996).

Se ha observado que es más factible dejar de fumar y permanecer abstinente si el fumador cuenta con una buena red de soporte social general. Una 
mejor estructura de apoyo (medida por índices de integración y estado civil) es un factor predictor de escaso riesgo de recaída. De la misma forma el éxito en el tratamiento parece estar inversamente relacionado con el nivel de interacciones sociales negativas. El tener una mayor red de apoyo social puede contribuir a crear un ambiente más manejable en general, disminuyendo los niveles de estrés y de forma indirecta la probabilidad de volver a fumar. (Glasgow, Klesges y O’Neill, 1986; Havassy, Hall y Wasserman, 1991; Carmody, 1992).

Por último, y en un sentido más amplio, a nivel institucional, comunitario y en los lugares de trabajo se pueden crear estrategias que ayuden a las personas a dejar de fumar y a permanecer abstinentes. La regulación del uso del tabaco en espacios públicos y de manera prioritaria en el ambiente laboral puede contribuir a que el exfumador perciba un ambiente favorecedor.

\section{PROCEDIMIENTOS DE PREVENCIÓN DE RECAÍDAS}

Las altas tasas de recaídas que encontramos tras los tratamientos para dejar de fumar nos hacen ver la importancia que tiene en toda intervención de este tipo el abordaje de las recaídas desde un primer momento. A continuación se exponen a modo de resumen las estrategias que se han mostrado más eficaces como procedimientos para el mantenimiento de la abstinencia.

\section{Entrenamiento en prevención de recaídas}

Es importante que forme parte del tratamiento desde su inicio y consiste en realizar un entrenamiento de habilidades con las que la persona sepa identificar cuáles son sus situaciones de alto riesgo y cómo debe afrontarlas (habilidades sociales, respuestas asertivas para resistir las presiones del entorno, etc.). También se han mostrado útiles las técnicas de reestructuración cognitiva para enseñar a manejar las atribuciones derrotistas si se produce un desliz así como para modificar las expectativas positivas del consumo(Hall, et.al. , 1984; Curry y Mc Bride, 1994; Echeburúa, 1994).

\section{Sesiones de refuerzo y contacto prolongado}

Se ha propuesto la utilización de sesiones de refuerzo una vez finalizado el tratamiento y cuando va a existir un periodo de tiempo en el que no va a haber contacto. En estas sesiones se tratarían de refrescar las estrategias enseñadas para enfrentarse al situacio- nes difíciles sin consumir y reforzar a los participantes sus esfuerzos individuales por mantener la abstinencia. (Curry y Mc Bride, 1994).

El prolongar el contacto tras el tiempo del tratamiento a través de intervenciones telefónicas, contacto por escrito o grupos de apoyo se ha mostrado como una estrategia eficaz (Brownell, et. al., 1986; Prochaska, et. al., 1993; US Public Health Service, 2000). Ossip-Klein y colaboradores (1991) encontraron mayores tasas de abstinencia en aquellas personas que tenían acceso a una línea telefónica en la que podían recibir asesoramiento sobre las estrategias a utilizar en caso de situaciones de alto riesgo. Estos resultados también se observaron en otro estudio que añadía al contacto telefónico la posibilidad de acudir a grupos de apoyo y llevar a cabo el tratamiento con un compañero (buddy system) (Gruder, et.al. , 1993).

\section{Otras intervenciones}

Los procedimientos para dejar de fumar también deben incluir el abordaje de ciertos factores que pueden precipitar el abandono de la abstinencia. En lo referido a la aparición de estados de ánimo negativos es importante que los programas incluyan la posibilidad de recibir asesoramiento así como de prescribir la medicación adecuada y en todo caso la valoración de la posibilidad de referir a la persona a un especialista. En cuanto a la aparición de síntomas de abstinencia muy intensos y/o prolongados en el tiempo se debería considerar la posibilidad de continuar con la medicación con la que inicialmente comenzó o añadir un nuevo fármaco de forma que la combinación de ambos reduzca la severidad de los síntomas (peje. utilización de parche de nicotina y bupropion). Estas intervenciones deben incluir estrategias para el control del peso insistiendo en la necesidad del ejercicio físico y de una dieta equilibrada aunque es recomendable fomentar la idea de que es normal ganar algo de peso al dejar de fumar evitando la utilización de dietas restrictivas. (US Public Health Service, 2000).

\section{CONCLUSIONES}

Las recaídas son parte del fenómeno de dejar de fumar. La mayoría de los fumadores quieren dejar de serlo y muchos de ellos lo intentan seriamente, algunos incluso siguiendo programas de tratamiento. Sin embargo, son pocos los que logran mantenerse abstinentes al cabo de un año. En consecuencia la recaída va a ser un condicionante importante para lograr disminuir significativamente la prevalencia de fumadores.

Diversos factores pueden determinar la recaída. Se puede intervenir fundamentalmente enseñando estra- 
tegias al fumador para afrontar las situaciones de riesgo y controlar los cambios emocionales aunque existen algunos factores, como los sociodemográficos, que son difícilmente modificables. Por otro lado, las medidas para controlar las variables ambientales que influyen en este proceso van a superar el análisis de la intervención sanitaria, siendo éstas en muchas ocasiones medidas reglamentarias que ya se han mostrado eficaces en otros países.

Existen aún interrogantes fundamentales sobre las variables que han sido objeto de análisis tanto en lo referente a su papel en el proceso como a la interrelación entre los diferentes factores.

\section{BIBLIOGRAFÍA}

AMERICAN PSYCHIATRIC ASSOCIATION (1994). Diagnostic and Statistical Manual of Mental Disorders, Fourth Edition, American Psychiatric Association, Washington, DC.

ANDA, R.F., WILLIAMSON, D.F., ESCOBEDO, L.G., MAST, F., GIOVINO, G.A. Y REMINGTON, P.L. (1990). Depression and the dynamics of smoking. Journal of American Medical Association 264(12):1541-1545

BAER, J.S. Y LICHTENSTEIN, E. (1988). Classification and prediction of smoking relapse episodes: An exploration of individual differences. Journal of Consulting and Clinical Psychology 56(1)104-110

BAER, J.S., KAMARCK, T., LICHTENSTEIN, E. Y RANSOM CC Jr. (1989). Prediction of smoking relapse: analyses of temptations and transgressions after initial cessation. Journal of Consulting and Clinical Psychology 57(5):623-627

BANDURA, A. (1986). Social Foundations of Thoughts and Actions. Prentice Hall: Englewood Cliff, NJ.

BECOÑA, E., LISTA, M.J. Y FROJAN, M.J.(1989). ¿Por qué los sujetos recaen en los tratamientos de dejar de fumar?. Un estudio exploratorio. Revista Española de Drogodependencias 14(1):29-37

BENOWITZ, N.L. (1983). The use of biological fluid samples in assessing tobacco smoke consumption. NIDA Research Monograph Series 48: 6-26

BENWELL, M.E., BALFOUR, D.J. Y ANDERSON, J.M. (1988). Evidence that tobacco smoking increases the density of (-)-[3H] nicotine binding sites in human brain. Journal of Neurchemistry. 50(4):1243-1247

BLISS, R.E., GARVEY, A.J., HEINOLD, J.W. Y HITCHCOCK, J.L. (1989). The influence of situation and coping on relapse crisis. Outcomes after smoking cessation. Journal of Consulting and Clinical Psychology 57(3): 443-449

BRANDON, T.H., TIFFANY, S.T., OBREMSKI, K.M. Y BAKER, T.B. (1990). Postcessation cigarette use: The process of relapse. Addictive Behaviors 15:105-114
BROWNELL, K.D., MARLATT, G.A, LICHTENSTEIN, E. Y WILSON, G.(1986). Understanding and preventing relapse. American Psychologist July: 765-781

CARMODY, T.P (1992). Preventing relapse in the treatment of nicotine addiction: Current issues and future directions. Journal of Psychoactive Drugs. 24(2):131-158

CASTILLO, R., Y MUSITU, G.(1992). Dimensiones psicosociales de la recaída en el consumo de tabaco: un análisis en fumadores y exfumadores. Adicciones 4(4):299-314

CENTERS FOR DISEASE CONTROL. (1991). Cigarette Smoking-attributable mortality and years of potential life lost: United States. MMWR Morb Mortal Wkly Rep 42:854-857

CHEN, Y., RENNIE, D.C., LOCKINGER, L.A. Y DOSMAN, J. (1998). Excessive weight concerns in ex smoking women. International Journal of Epidemilogy 27(5): 927-928

COHEN, S., LICHTENSTEIN, E., PROCHASCKA, J.O., GRITZ, E.R., CARR, C.R., ORLEANS, C.T., SCHOENBACH, V.J., BIENER, L., ABRAMS, D., ET.AL. (1989). Debunking myths about self-quitting. Evidence from 10 prospective studies of persons who attempt to quit smoking by themselves. American Psychology 44(11):1355-65.

COHEN, S. Y LICHTENSTEIN, E. (1990). Partner behaviors that support quitting smoking. Journal of Consulting and Clinical Psychology. 58(3): 304-309

COVEY, L.S., GLASSMAN, A.H. Y STETNER, F. (1998). Cigarette smoking and major depression. Journal of Addictive Diseases 17(1):35-46

COVEY, L.S., GLASSMAN, A.H., Y STETNER, F. (1997) Major depression following smoking cessation. American Journal of Psychiatry Feb;154(2):263-5

CUMMINGS, C., GORDON, J.R., Y MARLATT, G.A. (1980). Relapse: Prevention and prediction. En Miller WR (Ed).The addictive disorders:Treatment of alcoholism, drug abuse, smoking and obesity (pp.291-322). New York: Pergamon.

CURRY, S., Y MARLATT, G.A. (1985). Unaided quitters' strategies for coping with temptations to smoke. En Shiffman S y Wills TA (Eds.) Coping and Substance Use (pps.243-265). New York: Guilford Press

CURRY, S.J., WAGNER, E.H., CHEADLE, A., DIEHR, P., KOEPSELL, T., ET AL . (1993). Assessment of communitylevel influences on individuals' attitudes about cigarette smoking, alcohol use and consumption of dietary fat. American Journal of Preventive Medicine 9:78-84

CURRY, S.J. Y MCBRIDE, C.M. (1994) Relapse prevention for smoking cessation: Review and Evaluation of Concepts and Interventions. Annual Review of Public Health. 15: 345-366.

DROBES, D.J., MEIER, E.A. Y TIFFANY, S.T. (1994). Assessment of the effects of urges and negative affect on smokers' coping skills. Behavior Research Therapy. 32(1): 165-174. 
EDWARDS, N. Y SIMS-JONES, N. (1998). Smoking and smoking relapse during pregnancy and postpartum: results of a qualitative study. Birth 25(2):94-100

ECHEBURÚA, E. Y DE CORRAL, P. (1986). Predicción de la recaída en las conductas adictivas:estrategias de intervención. Drogalcohol 11(1):16-25.

ECHEBURÚA, E. (1994). Evaluación y tratamiento de los trastornos adictivos. Fundación Universidad Empresa.

FAUE, M., FOLEN, R.A., JAMES, M.L.C., Y NEEDELS, T. (1997). The trippler tobacco-cessation program: predictors for success and improved efficacy. Militar Medicine 162:445-449

FIORE, M.C., NOVOTRY, T.C., PIERCE, J.P., GIOBINO, G.A., HATZIANDREU, E.J., NEWCOMB, P.A., SURAWICZ, T.S. Y DAVIS, R.M. (1990). Methods used to quit smoking in the United States. Do cessation programs help? Journal of American Medical Association 1990; 263(20):2760-2765

GARVEY, A.J., BLISS, R.E., HITCHCOCK, J.L., HEINOLD, J.W. Y ROSNE, B. (1992). Predictors of smoking relapse among self-quitters: A report from the normative aging study. Addictive Behaviors 17:367-377

GILPIN ,E.A., PIERCE,A. Y FARKAS, J. (1997). Duration of smoking abstinence and success in quitting. Journal of the National Cancer Institute. 89(8): 572-576.

GLASGOW, R.E., KLESGES, R.C. Y O’NEILL, H.K. (1986). Programming social support for smoking modification: an extension and replication. Addictive Behaviors. 11(4):453-457

GLASGOW, R.E. Y LICHTENSTEIN, E. (1987). Long-term effects of behavioral smoking cessation interventions. Behavior Therapy 18:297-324.

GLASSMAN, A.H., HELZER, J.E., COVEY, L.S., COTTLER, L.B., STETNER, F., TIPP, J.E. Y JOHNSON, J. (1990). Smoking, smoking cessation and mayor depression. Journal of American Medical Association 264 (12) : $1546-1549$

GONZALEZ, J., VILLAR, F., BANEGAS, J.R., RODRIGUEZ, F., Y MARTIN J.M.(1997) Tendencia de la mortalidad atribuible al tabaquismo en España, 1978-1992: 600.000 muertes en quince años. Med Clin (Barc) 109:577-582

GRUDER, C.L., MERMELSTEIN, R.J., KIRKENDOL, S., HEDEKER, D., WONG S.C., SCHRECKENGOST, J., WARNECKE, R.B., BURZETTE, R. Y MILLER, T.Q. (1993). Effects of social support and relapse prevention training as adjuncts to a televised smoking-cessation intervention. Journal of Consulting and Clinical Psychology 61(1):113-120

GULLIVER, S.B., HYGHES, J.R., SOLOMON, L.J. Y DEY, A.N. (1995). An investigation of self-efficacy, partner support and daily stresses as predictors of relapse to smoking in self-quitters. Addiction 90:767-772

HALL, S.M., RUGG, D., TUNSTALL, C. Y JONES, R.T. (1984). Preventing relapse to cigarette smoking by behavioral skill training. Journal of Consulting and Clinical Psychology. 52(3): 372-382
HALPERN, M.T. Y CARNER, K.E.(1993) Motivations for smoking cessation: a comparison of successful quitters and failures. Journal of Substance Abuse 5(3):247-256

HALEY, N.J, AXELRAD, C.M. YTILTON, K.A. (1983). Validation of self-reported smoking behavior: biochemical analysis of cotinine and thocynate. American Journal of Public Health 73(10): 204-207

HAVASSY, B.E., HALL, S.M. Y WASSERMAN, D.A. (1991). Social support and relapse: commonalties among alcoholics, opiate users and cigarette smoking. Addictive Behaviors 16(5):235-246

HELLMAN, R., CUMMINGS, K.M., HAUGHEY, B.P., ZIELEZNY, M.A. Y O'SHEA, R.M. (1991). Predictors of attempting and succeeding at smoking cessation. Health Education Research 6(1): 77-86

HUGHES, J.R. (1994). Protacted withdrawal American Journal of Psychiatry 151:785-786

HUGHES, J.R. (1992). The nicotine withdrawal syndrome: A brief review and update. International Journal of Smoking Cessation 1:21-26

HUGHES, J.R. (1988). Clonidine, depression and smoking cessation. Journal of the American Medical Association 259:2901-2902

HULTQUIST, C.M., RAY, J.W.Y SADISH, W.R. (1997). How much weight gain occurs following smoking cessation? A comparison of weight gain using both continuous and point prevalence abstinence. Journal of Consulting and Clinical Psychology 65(2): 286-291

HYMOWITZ N, CUMMINGS KM, HYLAND A, LYNN W, PECHACEK Y HARTWELL, T.D. (1997). Predictors of smoking cessation in a cohort of adult smokers followed for five years. Tobacco Control 6(Suppl):S57-S62

JANZON, L., LINDELL, S.E., TRELL, E. Y LARME, P. (1981). Smoking habits and carboxyhaemoglobin. A crosssectional study on urban population of middle-aged men. Journal of Epidemiology and Community Health. 31:271-273

JARVIS, M.J., RUSSELL, M.A.H. Y SALOJEE, Y. (1980). Expired air carbon monoxide: a simple breath test of tobacco smoke intake. British Medical Journal 281: 484-485

JOHNSON, J.L., RATNER, P.A., BOTTORTOFF, J.L., HALL, W. Y DAHINTEN, S. (2000). Preventing smoking relapse in postpartum women. Nursing Research 49(1):4452

KAMARCK, T.W. Y LICHTENSTEIN, E. (1988). Program adherence and coping strategies as predictors of success in an smoking treatment program. Health Psychology. 7(6):557-74

KILLEN, J.D. Y FORTMANN, S.P. (1997). Craving is associated with smoking relapse: findings from three prospective studies. Experimental and Clincal Psychopharmacology 5(2):137-142

KLESGES, R.C., WINDERS, S.E., MEYERS, A.W., ECK, L.H., WARD, K.D.,

LANDO, H.A., PIERE, P.L., ROSKI J., MCGOVERN P.G. Y SCHMID L.A (1997). Promoting abstinence among relapsed chronic smokers: the effect of telephone 
support. American Journal of Public Health. 86(12): 1786-1790

LICHTENSTEIN, E., WEISS, S.M., HITCHCOCK, J.K., LEVETON, L.B., O'CONELL, K.A. Y PROCHASKA, J.O. (1986). Patterns of smoking relapse. Health Psychology 5 (Suppl):29-40

MARLATT, G.A. Y GORDON, J.R. (1980). Determinants of relapse: Implications for the maintenance of behavior change. In Davidson PO \& Davidson SM (Eds): Behavioral Medicine: Changing health lifestyles. (pp.410-452). Elmsford, NY: Pergamon

MARLATT, G.A. Y GORDON, J.R.(1985) Relapse prevention: maintenance strategies in addictive behavior change. New York: Guilford

MATHENY, K.B. Y WEATHERMAN, K.E. (1998). Predictors of smoking cessation and maintenance. Journal of Clinical Psychology 54(2): 223-235

MC BRIDE, C.M., CURRY, S.J., LANDO, H.A., PIRIE, P.L., GROTHAUS LCY NELSON JC. (1999). Prevention of relapse in women who quit smoking during pregnancy. American Journal of Public Health 89(5): 706-711

MERMELSTEIN, R.J. Y LICHTENSTEIN, E. (1983). Slips versus relapses in smoking cessation. Western Psychological Association paper meeting, San Francisco MERMELSTEIN, R.J., KARNATZ, T. Y REISCHMANN, S. (1992). Smoking. En PH Wilson (ed) Principles and practice of relapse prevention. 3:43-68. New York, NY:Gulford Press.

MEYERS, A.W., KLESGES, R.C., WINDERS, S.E., WARD, K.D., PETERSON, B.A. Y ECK, L.H .(1997). Are weight concerns predictive of smoking cessation?. A prospective analysis. Journal of Consulting and Clinical Psychology 65(3):448-452

MIZOUE, T., UEDA, R., TOKUI, N., HINO, Y. Y YOSHIMURA, T. (1998). Body mass decrease after initial gain following smoking cessation. International Journal of Epidemiology 27(6):984-988

MORENO, J.J. Y HERRERO, F.J. (2000). Factores asociados al éxito en programas para dejar de fumar. Adicciones 12(Suppl 1): 35-36

MULLEN, P.D., RICHARDSON, M.A., QUINN, V.P. Y ERSHOFF, D.H. (1997). Postpartum return to smoking: who is at risk and when. Health Promotion 11(5):323-330

NIDES, M.A., GONZALES, D., TASHKIN, D.P., RAKOS, R.F., MURRAY, R.P., BJORNSON-BENSON,W.M., LINDGREN,P.Y CONETT, J.E (1995). Predictors of initial smoking cessation and relapse though the first 2 years of the Lung Health Study. Journal of Consulting and Clinical Psychology. 63(1):60-69.

NIETO, M.L. (2000). Epidemiología del Tabaquismo. En F. Carrión (Coord). Tabaquismo/Tabaquisme. Una perspectiva desde la Comunidad Valenciana. (pp.29-36). Valencia: Conselleria de Sanitat

OCKENE, J.K., BENFARI, R.C., NUTTALL, R.L., HURWITZ, I. Y OCKENE, I.S. (1982). Relationship of psychosocial factors to smoking behavior change in an intervention program. Preventive Medicine 11(1):13-28
OCKENE, J.K., EMMONS, K.M., MERMELSTEIN, R.J., PERKINS, K.A., BONOLLO, D.S., VOORHEES, C.C. Y HOLLIS, J.F. (2000). Relapse and maintenance issues for smoking cessation Health Psychology 19 (1 Suppl): 17-31

O'CONELL, K.A. Y MARTIN, E.J. (1987). Highly tempting situations associated with abstinence, temporary lapse and relapse among participants in smoking cessation programs. Journal of Consulting and Clinical Psychology 55:367-371

O'CONELL, K.A. (1990). Smoking cessation: research on relapse crisis. Annual Review of Nursing Research 8:83-100

O'CONELL, K.A., GERKOVICH, M.M., COOK, M.R., SHIFFMAN, S., HICKOX, M. Y KAKOLEWSKI, K.E. (1998). Coping in real time: using Ecological Momentary Assessment techniques to assess coping with the urge to smoke. Research ins Nursing and Health 21(6):487497

O'HARA, P., CONETT, J.E., LEE, W.W., NIDES, M., MURRAY, R. Y WISE, R. (1998). Early and weight gain following smoking cessation in the Lung Health Study. American Journal of Epidemiology 1;148(9):821-830

OSSIP-KLEIN, D.J., BIGELOW, G., PARKER, S., CURRY, S., HALL, S.M. Y KIRKLAND, S. (1986). Task Force 1: Classification and assessment of smoking behavior. Health Psychology 5: 3-11

OSSIP-KLEIN, D.J., GIOVINO, G.A., MEGAHED, N., BLACK, P.M., EMONT, S.L., et.al. (1991). Effects of a smoker's hotline: results of a 10-county self-help trial. Journal of Consulting and Clinical Psychology. 59(2):325-332

PETO, R., LOPEZ, A.D., BOREHAM, J., TUN, M. Y HEALTH, C.J.R. (1992). Mortality from tobacco in developed countries: indirect estimation from national vital statistical. Lancet, 339(8804):1268-1278

PIASECKI, T.M., FIORE, M.C. Y BAKER, T.B.(1998). Profiles in discouragement: two studies of variability in the time course of smoking withdrawal symptoms. Journal of Abnormal Psychology 107(2):238-251

PROCHASKA, J.O. Y DI CLEMENTE, C.C. (1983). Stages and process of self-change of smoking: Toward and integrative model of change. Journal of Consulting and Clinical Psychology 51:390-395

PROCHASKA, J.O., DI CLEMENTE, C.C., VELICER, W.F. Y ROSSI, J.S. (1993). Standarized, individualized, interactive and personalized: Self-help programs for smoking cessation. Health Psychology 12:399-405

RATNER, P.A., JOHNSON, J.L., BOTTORFF, J.L., DAHINTEN, S. Y HALL, W. (2000). Twelve-month follow-up of a smoking relapse prevention intervention for postpartum women. Addictive Behaviors 25(1):81-92

ROSE, JE. (1996). Nicotine addiction and treatment. Ann Rev Med 47:493-507

SALVADOR, T. (1996). Tabaquismo. Madrid: Aguilar

SÁNCHEZ, L., CARRERAS, J.M. Y MALDONADO, B. (1998). Efficiency of the smoking multicomponent treatment in a neumologic unit. Results and predictors factors of 
success. The European Respiratory Journal 12(Suppl 28): $82 \mathrm{~S}$

SECKER-WALKER, R.H., SOLLOMON, L.J., FLYNN, B.S. Y SKELLY, J.M.. (1995). Smoking relapse prevention counselling during prenatal and early postnatal care. American Journal of Preventive Medicine 11(2): 8693

SHIFFMAN, S.(1982) Relapse following smoking cessation: A situational analysis. Journal of Consulting and Clinical Psychology 50:71-86

SHIFFMAN, S. (1986) A cluster analytic classification of smoking relapse episodes. Addictive Behaviors 11:295307.

SHIFFMAN, S., SHUMAKER, S.A., ABRAMS, D.B., COHEN, S., GARVEY, A., GRUNBERG, E.N. Y SWAN, G.E.(1986) Models of smoking relapse. Health Psychology 5 (Suppl): 13-27

SHIFFMAN, S., PATY, J.A., GNYS, M., KASSEL, JA., Y HICKOX, M.(1996a). First lapses to smoking: withinsubjects analysis of real-time reports. Journal of Consulting and Clinical Psychology. 64(2): 366-379.

SHIFFMAN, S., HICKOX, M., PATY, JA., GNYS, M., KASSEL, JD. Y RICHARDS DS, T.J (1996b). Progression from a smoking lapse to relapse: prediction from abstinence violation effects, nicotine dependence, and lapse characteristics. Journal of Consulting and Clinical Psychology 64(5): 993-1002.

SHIFFMAN, S., HICKCOX, M., PATY, J., GNYS, M., RICHARDS, T. Y KASSEL, J.D.(1997). Individual differences in the context of smoking relapse episodes. Addictive Behaviors 22(6): 797-811

SOLOMON, R.L. Y CORBIT, J.D. (1973). An opponentprocess theory of motivation: II. Cigarette addiction. Journal of Abnormal Psychology. 81: 158-171

STEAD, L.F., Y LANACASTER T. (2000). Group behaviour therapy programmes for smoking cessation (Cochrane
Review). En: The Cochrane Library, Issue 3. Oxford: Update Software.

SUTTON, S.R. (1992). Are "risky" situations really risky? Review and critique of the situational approach to smoking relapse. Journal of Smoking-Related Disorders 3: $79-84$

SWAN, G.E., WARD, M.M. Y JACK, L.M. (1996). Abstinence effects as predictors of 28-day relapse in smokers. Addictive Behaviors 21(4):481-90

U.S DEPARTMENT OF HEALTH AND HUMAN SERVICES.(1990). The Health Benefits of Smoking Cessation: A Report of Surgeon General. U.S Department of Health and Human Services: Rockville MD. DHHS Publication No (CDC)90-8416

U.S. PUBLIC HEALTH SERVICE (2000). A clinical practice guideline for treating tobacco use and dependence. JAMA 83(24):3244-3254

VÁZQUEZ, F. Y BECOÑA, E. (1998). ¿El hábito de fumar tiene relación con la depresión?. Psicothema 10(2):229-239

VELICER, W.F., PROCHASKA, J.O, ROSSI, J.S. Y SNOW, M.G. (1992). Assessing outcome in smoking cessation studies. Psychological Bulletin 111(1): 23-41

WALD, N.J. Y HACKSHAW, A.K.(1996) Cigarette Smoking: an epidemiological overview. British Medical Bulletin 52(1):3-11

WISE, R.A.(1988). The neurobiology of craving:Implications for the understanding and treatment of addiction. Journal of Abnormal Psychology 97:118-132

WITLOCK, E.P., VOGT, T.M., HOLLIS, F.J. Y LICHTENSTEIN, E. (1997). Does gender affect response to a brief clinic-based smoking intervention? American Journal of Preventive Medicine 13(3):159-166.

WOJCIK, J.V. (1988). Social learning predictors of the avoidance of smoking relapse. Addictive Behaviors $13: 177-180$ 
